\title{
Synthesis of Photoconducting Non-linear Optical Co-Polymer of 2-Methacryloyl-1-(4'-nitro-4-azo-1'Phenyl) Salicylic acid With Methylacrylate
}

\author{
*Dipak Kumar Mukhopadhyay \\ Institute of Science \& Technology, Chandrakona Town, West Bengal, 721201, India
}

\begin{abstract}
The azo dye namely 4-nitro phenyl azo salicylic acid was synthesized and characterized by $I R, U V$ and NMR spectroscopy. The monomer was synthesized by the reaction of azo dye namely 4-nitrophenyl azo salicylic acid and methacryloyl chloride in the solvent medium of THF under reflux. Lastly the monomer 2methacryloyl-1- (4'-nitro-4-azo-1'-phenyl)salicylic acid was co-polymerzed with methylacrylate in the solvent medium of DMF at $110^{\circ} \mathrm{c}$. All the monomers and co-polymer were characterized by IR,UV and NMR spectroscopy. The polymer has good photoconducting property. Moreover the non-lincar optical polymer can be used as photorefractive system. The polymer has good thermal stability indicating occurance of strong inter and intramolecular dipolar interactions Originated by the presence of high charge delocalization in the macromolecular side chain.
\end{abstract}

Key Words: 4-nitrophenyl azo salicylic acid, 2-methacryloyl-1-(4'-nitro-4-azo-1'-phenyl) salicylic acid, copolymer, radical polymerization.

\section{Introduction}

Non-linear optical chromophore functionalized polymers have greater advantages over the guest-host systems. In guest-host system, the NLO chromophore possessing high first hyperpolarizabilityare dispersed in an inert amorphous polymer matrix of high optical transparency.However, guest-host systems are found to be of low NLO activity. Orientatinal relaxation, poor solubility, thermal and environmental instability of chromophores in the host polymer matrix are major reasons behind low NLO activity.In order to improve the above mentioned drawbacks, various designing methods were adopted to attach NLO chromophore functionalized side chain and main chain polymers, chromophoric main chain polymers with NLO-active side chains, crosslinked NLO polymers and ferroelectric polymers.

In this article electro-optic non-linear optical co-polymer are synthesized. In NLO-chromophore functionalized polymers, the NLO chromophore are covalently bonded to the polymer backbone via chemical modifications. The advantages of NLO-chromophore functionalized polymers over guest-host systems are : high concentration of NLO chromophores can be introduced to the polymer backbone, absence of phase separation, greater stability towards orientational relaxation and they can be processed into thin films. This class of polymers usually have polystyrene,poly(methyl methacrylate) and polyethylene type backbone.

Theelectro-optic polymer, poly(2-methacryloyl-1-(4'-nitro-4-azo-1'-phenyl) salicylic acid-comethylacrylate)was synthesized via radical polymerization.It is a copolymer of methylacrylate and azo dyesubstituted methacrylate. Azo- substituted polymers are receiving greater attention because of their potential applications in the field of non-liner optics, optical storage media, etcAzo polymers have good transparency and high non- linear optical susceptibility. The presence of azo group in the chromophore results in extended conjugation. The extended conjugation between doner and acceptor groups is responsible for enhanced NLO properties.

The synthesized co- polymer was characterized by IR, UV and NMRspectroscopy . The co- polymer system showed an increased stability compared to guest- host system. The co-polymer has good thermal stability. Further the electro- optic coefficient of the co- polymer can be measured.

\subsection{Synthesis of 4-nitrophenyl azo salicylic acid :-}

\section{Experimental:}

A mixture of $7 \mathrm{~g}$ of 4 - nitro aniline, $20 \mathrm{ml}$ of concentrated hydrochloric acid and $40 \mathrm{ml}$ of water was prepared in a $500 \mathrm{ml}$ beaker. This was heated until the solution was completely clear. The solution was cooled to $5^{0} \mathrm{c}$ with adding $40 \mathrm{~g}$ of ice. Another mixture of $3.5 \mathrm{~g}$ sodium nitrite in $10 \mathrm{ml}$ water was prepared in a $100 \mathrm{ml}$ beaker. Sodium nitrite solution was added into above prepared solution keeping the temperature below $5^{0} \mathrm{c} .7 \mathrm{~g}$ of salicylic acid in $30 \mathrm{ml} 20 \%$ sodium carbonate was mixed and the mixture was cooled to $5^{0} \mathrm{c}$. The diazo solution was poured into the solution of salicylic acid at $5^{0} \mathrm{c}$. The mixture was made alkaline by adding $20 \%$ of sodium carbonate solution. The reaction mixture was stirred at $5^{\circ} \mathrm{c}$ for $2 \mathrm{~h}$. after that $10 \%$ sodium chloride 
solution was added and heated the mixture at $45-50^{\circ} \mathrm{c}$ till the salt dissolved completely. The mixture was cooled and stirred at room temperature for $1 \mathrm{~h}$ till the precipitation of the dye was completed. The dye was filtered and dried under vacuum. The synthesizeddye was characterized by IR, UV and NMR spectroscopy.

\subsection{Synthesis of 2-methacryloyl-1-(4'-nitro-4-azo-1'-phenyl) Salicylic acid:}

4-nitrophenyl azosalicylic acid $(2.87 \mathrm{~g}, 10 \mathrm{~m} \mathrm{~mol})$ was dissolved in $25 \mathrm{ml}$ of dry THF was cooled to $0^{\circ} \mathrm{c}$ under nitrogen. To this solution pyridine $(0.1 \mathrm{~g}, 1.26 \mathrm{~m} \mathrm{~mol})$ and methacryloyl chloride $(1.05 \mathrm{~g}, 10 \mathrm{~m}$ mol) were added dropwise simultaneously. The reaction was carried out for $40 \mathrm{~h}$ at reflux temperature. The reaction mixture was cooled and filtered. The solid obtained was purified by recrystallization from ethanol. The obtained monomer was characterized by IR, UV and NMR Spectroscopy.

\subsection{Polymerization:}

2-methacryloyl-1-(4'-nitro-4-azo-1'-phenyl) salicylic acid $(1.42 \mathrm{~g}, 4 \mathrm{~m} \mathrm{~mol})$, methyl acrylate $(0.35 \mathrm{~g}, 4 \mathrm{~m}$ mol) and benzoyl peroxide $(2.21 \mathrm{~g}, 10 \mathrm{~m} \mathrm{~mol})$ were dissolved in $40 \mathrm{ml}$ of dry DMF. The reaction was carried out at $110^{\circ} \mathrm{c}$ for $80 \mathrm{~h}$ under nitrogen. The resulting solid was dissolved in DMF and reprecipitated from methanol. The polymer was collected by filtration, dried under vacuum and stored. The polymer was characterized by IR, UV and NMR spectroscopy.

\section{Result and Discussion:}

Azo polymers are of two types : side chain and main chain azo polymers, based on the position of the azo group. The presence of azo group in the main chain and side chain polymers provides long conjugation between doner and acceptor group. Azo polymers generally have polystyrene, polymethacrylates, polyesters, polysiloxanes, polysilanes etc. as polymer backbones. They are usually synthesized by polymerization and copolymerization of styrene, methacrylates, methylacrylates, esters, silanes, siloxanes etc. with azo substituted monomers. The preparation of the co-polymer with methylacrylate is multi-step process. At first the azo dye was prepared as follows: 4-nitro aniline was diazotized using sodium nitrite and hydrochloric acid at $\mathrm{o}^{\circ} \mathrm{c}-5^{\circ} \mathrm{c}$ Then the required dye was synthesized by coupling thediazo salt with salicylic acid. The synthetic route of the azo dye was illustrated in scheme-1.

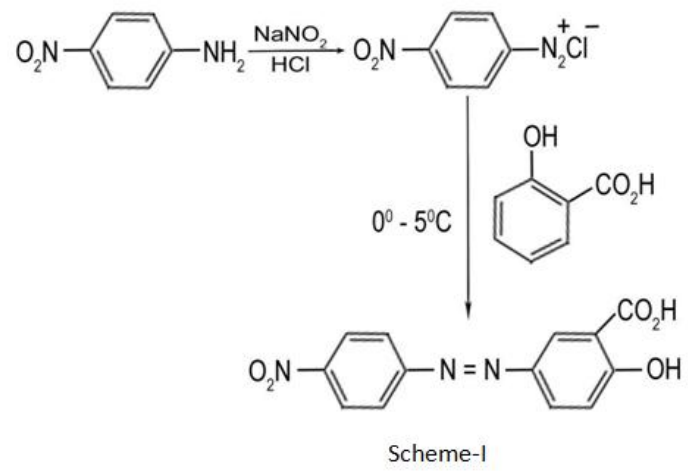

Scheme-I

Secondly the NLO monomer was synthesized by the reaction of azo dye namely 4-nitro phenylazo salicylic acid with methacryloyl chloride under THF in appropriate ratio. The synthetic route for the preparation of NLO monomer was illustrated in scheme - II

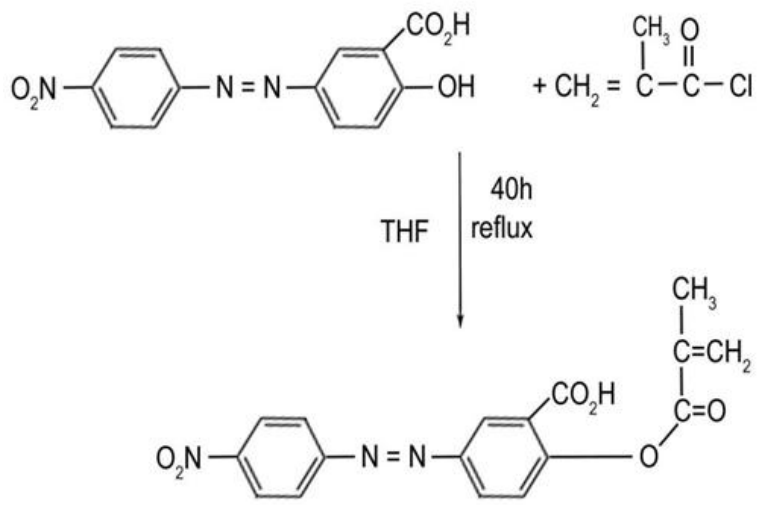

Scheme- II 
Lastly the co-polymer was synthesized by the radical polymerization of the NLO monomer namely 2methacryloyl-1- (4'-nitro-4-azo-1'phenyl) salicylic acid with methyl acrylate by using benzoyl peroxide as a thermal initiator. The reaction was carried out in dry DMF at $110^{\circ} \mathrm{c}$ for $80 \mathrm{~h}$. The crude polymeric product was dissolved in DMF and precipitated by the addition of methanol. The polymer was obtained in acceptable yields. UV, IR and NMR spectra revealed the successful preparation of the polymer. The synthetic route for the preparation of the polymer was illustrated in scheme- III.<smiles>C=CC(=O)Oc1ccc(N=Nc2ccc([N+](=O)[O-])cc2)cc1C(=O)O</smiles><smiles>[Y]C(C)(C)[C@H](CC(C)(C)C(C)(C)C(C)(C)C(=O)Oc1ccc(N=Nc2ccc([N+](=O)[O-])cc2)cc1C(=O)O)C(=O)OC</smiles>

\section{Conclusion:}

Poly (2-methacryloyl -1-(4'-nitro-4-azo-1'-phenyl) salicylic acid -co- methylacrylate) was synthesized via radical initiated polymerization. It was a co-polymer of 2- methacryloyl-1- (4'-nitro-4-azo-1'-phenyl) salicylic acid and methyl acrylate. The structure of the polymer was confirmed by FT-IR, UV and NMR analysis. The polymer has good NLO and thermal properties. The polymer can be doped with $\mathrm{C}_{60}$ to enhance the photoconductivity.

\section{References}

[1]. C. Barrett, B. Chowdhury, A. Natansohn, P. Rochon, Macromolecules 31 (1998) 4845.

[2]. J. Park, T. J. Marks, Chem Mater. 2 (1990) 229.

[3]. D.M.Burland, R. D. Miller, C.A. Walsh, Chem.Rev. 94 (1984) 31.

[4]. Y. Chen, B. Zhang, F. Wang, Opt. Commun. 228 (2003) 341.

[5]. S. Song, S. J. Lee, B. R. Cho, Chem. Mater. 11 (1999) 1406.

[6]. $\quad$ C. Xu, B.Wu, M.W. Becker, L.R. Dalton, Chem. Mater. 5 (1993) 1439.

[7]. T. K. Lim, S.H. Hong, M.Y. Jeong, G. J. Lee, Macromolecules 32 (1999) 7051.

[8]. D. H. Choi, W. M. K. P. Wijekoon, H. M. Kim, P.N. Prasad, Chem. Mater. 6 (1994) 234

[9]. C. B.Yoon, K.J. Moon, H.K. Shira, Macromolecules. 29 (1996) 5754.

[10]. L. Brzozowski, E. H. Sargent, J.Mater. Sci. Mater. Elec. 12 (2001) 483.

[11]. S. Xie, A. Natansohn, P. Rochon, Chem. Mater. 5 (1993) 403.

[12]. J.H.Lee, K.S. Lee, Bull. Korean Chem. Soc. 21 (2000) 847.

[13]. L. Angiolini, T. Benelli, L. Giorgini, E. Salatelli, Polymer 46 (2005) 2424

[14]. L. Angiolini, D. Caretti, L. Giorgini, E. Salatelli, Polymer 42 (2001) 4005.

[15]. W. You, D. Wang, Q. Wang, L. Yu, Macromolecules 35 (2002) 4636.

[16]. J. Sohn, J. Hwang, S. Y. Park, G. J. Lee, Jpn. J. Appl. Phys. 40 (2001) 3301

[17]. H. Moon, J. Hwang, N. Kim, S.Y. Park, Macromokcules 33 (2000) 5116.

[18]. J. Hwang, J. Sohn, S.Y .Park, Macromolecules 36 (2003) 7970.

[19]. K. Meerholz, B. L. Volodin, Sandalphon, B. Kippelen, N. Peyghambarian, Nature 37 (1994) 479.

[20]. O. Ostroverkhova, K. D. Singer, J. Appl. Phys. 92 (2002) 1727.

[21]. C. J. Huang, Y. K. Su, .S.L. Wu, Mater. Chem. Phys. 84 (2004) 146

[22]. J. Hwang, J. Sohn, J. K. Lee, J. H. Lee, J. S. Chang, G. J. Lee, S. Y. Park, Macromolecules 34 (2001) 4656. 
[23]. E. Hattemer, R. Zentel, E. Mecher, K. Meerholz, Macromolecules 33 (2000) 1972

[24]. B. Kippelen, F. Meyers, N. Peyghambarian, S. R. Marder, J. Am. Chem. Soc. 119 (1997) 4559.

[25]. A. Gunnet-Jepsen, C. L. Thompson, R. J. Twieg, W. E. Moerner, Appl. Phys. Lett. 70 (1997) 1515

IOSR Journal of Applied Chemistry (IOSR-JAC) is UGC approved Journal with Sl. No. 4031, Journal no. 44190.

Dipak Kumar Mukhopadhyay. "Synthesis of Photoconducting Non-linear Optical Co-Polymer of 2-

Methacryloyl-1-(4'-nitro-4-azo-1'Phenyl) Salicylic acid With Methylacrylate." IOSR Journal of Applied Chemistry (IOSR-JAC) 10.07 (2017): 07-10. 